\title{
Exclusive dealing with imperfect downstream competition
}

\author{
Jose Miguel Abito* and Julian Wright ${ }^{\dagger}$
}

September 2006

\begin{abstract}
The existing literature on exclusive dealing is extended to take into account that buyers signing exclusive deals are typically competing firms that are differentiated from the perspective of their customers. We show, provided such downstream firms are not too differentiated or provided upstream firms can compete in two-part tariffs, exclusive dealing forecloses entry to a more efficient rival. An established upstream firm and competing downstream firms raise their joint profit by signing exclusive deals to protect the industry from upstream competition. Naked exclusion arises despite the Chicago School logic that buyers only sign contracts that make themselves (jointly) better off.
\end{abstract}

\section{Introduction}

The "Chicago School" defence of exclusive dealing (Posner, 1976 and Bork, 1978) remains highly influential despite the works of Rasmusen et al. (1991), Bernheim and Whinston (1998), and Segal and Whinston (2000) among others, which show that exclusive deals can be harmful. Part of its success lies in the simplicity of its argument. The argument goes that buyers will not accept exclusive deals that prevent competition since by construction, such contracts will lower the total surplus available to buyers. This reflects the deadweight loss of monopoly. Unless exclusive contracts provide some efficiency benefit,

\footnotetext{
${ }^{*}$ University of Toulouse.

$\dagger$ Corresponding author. National University of Singapore. email jwright@nus.edu.sg. We benefited from comments from seminar participants at the 2006 EARIE meetings, the National University of Singapore, and especially the suggestions of two anonymous referees and our editor. Any errors are exclusively our own.
} 
buyers will obtain greater surplus rejecting exclusive deals, taking advantage of competition between suppliers instead. However, this logic, like much of the literature which has followed it, either assumes buyers are final consumers or that their interests are aligned with final consumers. Once it is recognized that buyers are in fact almost always competing downstream firms ${ }^{1}$, the Chicago School logic can have a very different implication. Since industry profit under monopoly is generally greater than under competition, downstream firms will often be willing to sign exclusive contracts to limit competition by preventing efficient entry. This raises the joint profit of the incumbent and downstream competitors, and so the "surplus" that is available to the firms for signing contracts.

This paper presents a model of exclusive contracts that illustrates this logic by taking into account that buyers are firms that compete downstream, albeit imperfectly. The model we present follows Segal and Whinston (2000, Section IV) and Fumagalli and Motta (2006), and assumes the entrant produces a good identical to the incumbent's but at a lower marginal cost. When the fixed costs of entry are high, so that the entrant requires more than one downstream firm to profitably enter, exclusion arises as the unique outcome when the joint profit of the incumbent and downstream firms under monopoly exceed the profit the downstream firms can obtain from buying at the competitive level. This happens provided downstream competition is sufficiently strong. On the other hand, when downstream competition is limited, profits under monopoly are limited by double marginalization so that downstream firms do better not signing contracts and buying from a competitive upstream market. These results assume the incumbent can only offer simultaneous and non-discriminatory exclusive contracts, and upstream firms sell to downstream firms using linear wholesale prices. Exclusion becomes easier with discriminatory or sequential exclusive contracts (so that buyers' interests can be divided), or when upstream firms sell to downstream firms using two-part tariffs (so that double marginalization does not limit the profit that can be obtained under exclusion).

When an entrant only needs one downstream firm to cover its fixed costs of entry, discriminatory or sequential contracts no longer play any special role. Fumagalli and Motta analyze this case, showing entry arises naturally with intense downstream competition. They find that the demand of a single downstream firm can be large enough to cover the entrant's fixed costs since final consumers will switch

\footnotetext{
${ }^{1}$ Fumagalli and Motta (2006) note this is true in both historical and more recent cases.
} 
to the cheaper downstream firm. They argue that intense downstream competition can eliminate the incumbent's ability to exclude even if it can offer discriminatory or sequential contracts. We find the opposite result. With intense (but not perfect) competition, downstream firms are left with almost no profits in equilibrium. The incumbent and its buyers can do better by signing exclusive deals which prevent the entrant from competing, thereby sharing the resulting monopoly profits. This is true even if the cost of entry is arbitrarily small, even if the entrant's innovation is almost drastic (implying a large cost advantage), and even if the upstream firms are limited to using linear wholesale prices to sell to downstream firms.

More generally, exclusion will arise whenever a single downstream firm's share of the joint profits under monopoly is greater than the profit the firm can obtain by deviating and buying at the competitive level. With linear wholesale pricing, we find a unique exclusion equilibrium when intrabrand competition is sufficiently strong and a unique entry equilibrium when downstream competition is sufficiently weak. Thus, we find the opposite result to that of Fumagalli and Motta, who argue that exclusion is more likely when downstream competition is weak. Moreover, when wholesale pricing is allowed to be non-linear, we find exclusion is the unique equilibrium regardless of the degree of downstream competition. This reflects the absence of a double marginalization effect with two-part tariffs.

The difference in implications of our model and that of Fumagalli and Motta can be explained by analyzing what happens when a buyer deviates. In their model, in which buyers face a small fixed cost of staying active, buyers that sign are forced to exit the industry when a deviating buyer does not sign since they cannot make any profit in the subsequent competition game. This makes the deviating buyer a monopolist, allowing it to enjoy corresponding monopoly profits. Although the deviating buyer can also gain the entire downstream market when buyers are strong competitors in our model, it cannot earn high profits due to the competitive pressure exerted by the incumbent's buyer(s). We think assuming the downstream buyers are always active is the more reasonable of the two approaches. It is in the interests of the incumbent for it to have an active buyer (so as to deter entry), and so if buyers really do face small fixed costs of remaining active, the incumbent would commit to pay these costs as part of its exclusive contract.

The other difference with the model of Fumagalli and Motta is that we allow for downstream compe- 
tition to be imperfect. Even if their assumption of a fixed cost to buyers of remaining active is dropped (as they do in their Section III), we show their resulting equilibrium analysis is still not robust to allowing some differentiation between downstream firms. When both downstream firms are active, they note both entry and exclusion equilibria are possible. This reflects that the incumbent's wholesale price to its signed buyer is indeterminant, given it does not expect to sell anything in equilibrium competing against the more efficient rival. Allowing for a little bit of differentiation between downstream firms provides a way of selecting from this multiplicity of equilibria, indicating that it is the exclusion equilibrium that is selected.

Simpson and Wickelgren (2004) also analyze exclusive dealing with limited downstream competition amongst buyers. They too find a unique exclusion equilibrium with sufficiently strong downstream competition. Their model differs from ours since they adopt the Rasmusen et al. (1991) cost specification in which the incumbent and entrant have the same average cost function, whereas we follow Fumagalli and Motta in this regard. Simpson and Wickelgren's cost specification makes exclusion easier since the entrant cannot undercut the incumbent in equilibrium, and obtains no profit from entry. At the same time, exclusion is generally harder in their framework because they allow buyers to renegotiate their contracts. Another difference between our paper and theirs is that we allow for linear and non-linear wholesale pricing, again following Fumagalli and Motta. In our analysis, the critical degree of downstream competition necessary to support exclusion depends on the fixed cost of entry, the cost advantage of the entrant, and whether wholesale prices are linear or non-linear. We map out these relationships, showing that non-monotonic relationships can exist for certain parameter ranges.

The rest of the paper proceeds as follows. Section 2 presents the model we use. Section 3 establishes results with linear wholesale pricing, while Section 4 establishes results with two-part tariffs. Section 5 briefly concludes.

\section{The model}

In this section, we extend Fumagalli and Motta (2006) to the case in which exclusive contracts are between an upstream incumbent and differentiated downstream buyers that themselves compete for final consumers. For the sake of convenience we will refer to upstream firms as manufacturers and downstream 
firms as retailers, although obviously the application of the model is much more general. The incumbent manufacturer (denoted $I$ ) and the potential entrant (denoted $E$ ) produce an identical product. The entrant's advantage is that it enjoys lower marginal costs of production. Specifically, we follow Segal and Whinston (2000, Section IV) and Fumagalli and Motta by assuming the incumbent has marginal cost of production $c_{I}$, the more efficient entrant has marginal $\operatorname{cost} c_{E}<c_{I}$, but faces a fixed cost of entry equal to $F>0$. Two differentiated retailers 1 and 2 compete in prices to sell this product, and there is no cost to retailing.

The incumbent proposes exclusive contracts to the two retailers in the initial stage (or stages in the case of sequential offers), which the retailers either accept or reject. As in the existing literature on naked exclusion, this exclusive contract involves the incumbent offering some fixed compensation $x$ to retailers in return for them agreeing to purchase exclusively from the incumbent. Let $S$ denote the number of retailers that sign the contract $(S=0,1$, or 2$)$. After observing the retailers' decisions on whether to sign these contracts, the entrant makes its entry decision in stage 2 .

In the third stage, the manufacturer(s) make offers to each available retailer. Like Fumagalli and Motta, we consider both the case they are restricted to offer linear wholesale prices and when they are able to offer two-part tariffs. The latter are particularly relevant here, since with limited downstream competition double marginalization effects make two-part tariffs more desirable. The details of these offers are assumed to be not publicly observable, so that retailer $j$ does not observe the details of any offer retailer $i$ receives. ${ }^{2}$ We follow the existing literature by assuming that in the face of competition with the entrant, the incumbent is able to discriminate between retailers that have signed the exclusive contract and those that have not. In addition, we assume retailers' beliefs (conjectures about the contracts offered to their rival) are passive except that whenever a retailer receives an off-equilibrium offer from a manufacturer, it assumes the rival retailer also receives the same offer from the manufacturer in the case that the retailers have identical options (that is, whether they are able to buy from just the incumbent, or from either the

\footnotetext{
${ }^{2}$ Katz (1991) argues such offers are more realistic than those that are publicly observed. This assumption also allows us to simplify our presentation of the case with two-part tariffs so as to obtain results for general demand specifications. With linear wholesale pricing, allowing offers to be publicly observed does not change any results except that in Proposition 4 , the range of parameter values for which unique exclusion arises is slightly wider than the range characterized in this paper. The details are provided in a separate technical appendix, available on the corresponding author's website.
} 
incumbent or entrant). As noted by Rey and Vergé (2004), this solves the commitment problem which otherwise plagues the analysis when a single upstream firm sells to two competing downstream firms using two-part tariffs under unobservable contracts - the case of the $S=2$ subgame in Section 4 .

In the final stage, retailer $i$ competes for consumers by setting a price $p_{i}$. The incumbent's, entrant's and retailer $i$ 's profit (gross of any compensation payments) in the case $S=k$ retailers sign an exclusive contract in stage 1 are denoted $\Pi_{I \mid S=k}, \Pi_{E \mid S=k}$ and $\pi_{i \mid S=k}$ respectively.

We make the standard assumption that the entrant's innovation is non-drastic. We measure the entrant's cost advantage by $\theta$, where $c_{I}=\theta p^{m}\left(c_{E}\right)+(1-\theta) c_{E}$ and $p^{m}\left(c_{E}\right)$ is the monopoly price for the industry when marginal $\operatorname{cost}$ is $c_{E}$. Thus, $\theta=0$ implies the entrant has no cost advantage and $\theta=1$ implies the entrant's cost advantage is (just) drastic. We assume $0<\theta<1$ throughout. We also assume that the entrant's fixed costs $F$ is low enough for entry to be profitable in the absence of exclusive deals - otherwise, exclusive deals have no role to play since entry will never occur. To avoid open-set problems in defining equilibria, we adopt the tie-break rule that when retailers are indifferent between signing an exclusive contract or not, they sign the contract, and that retailers that do not sign buy from the more efficient manufacturer when indifferent.

Where we make use of a specific model of demand, it is taken to be defined by the standard representative consumer model (Bowley, 1924), where consumers' utility from the two retailers is defined by

$$
U\left(q_{1}, q_{2}\right)=\alpha\left(q_{1}+q_{2}\right)-\frac{\beta\left(q_{1}^{2}+q_{2}^{2}+2 \gamma q_{1} q_{2}\right)}{2},
$$

and $q_{i}$ is the amount consumed at retailer $i$. This provides a convenient way to parameterize the degree of competition between retailers. Specifically, inverse demand functions are given by $p_{i}=\alpha-\beta\left(q_{i}+\gamma q_{j}\right)$, where $0 \leq \gamma<1$ serves as a measure of the degree of differentiation, and $\alpha>c_{I}$. The higher $\gamma$ is, the more homogenous are the two retailers.

When the prices of the two retailers are sufficiently close, both will enjoy positive demands. However, when the prices of the two retailers strongly diverge, the higher priced retailer will receive no demand, while the lower priced retailer captures the entire market. Specifically, for any price $p_{j}$, when $p_{i} \geq$ $\alpha(1-\gamma)+\gamma p_{j}$, firm $i$ receives no demand, while for $0<p_{i} \leq\left(-\alpha(1-\gamma)+p_{j}\right) / \gamma$, firm $i$ captures the 
entire market. Thus, firm $i$ 's demand function is

$$
\begin{aligned}
q_{i} & =\frac{\alpha(1-\gamma)-p_{i}+\gamma p_{j}}{\beta\left(1-\gamma^{2}\right)} & \text { if } & & \frac{-\alpha(1-\gamma)+p_{j}}{\gamma}<p_{i}<\alpha(1-\gamma)+\gamma p_{j} \\
& =\frac{\alpha-p_{i}}{\beta} & \text { if } & & 0<p_{i} \leq \frac{-\alpha(1-\gamma)+p_{j}}{\gamma} \\
& =0 & \text { if } & & p_{i} \geq \alpha(1-\gamma)+\gamma p_{j} .
\end{aligned}
$$

We will make use of this model of demand in the next section, which explores how the degree of differentiation between retailers determines whether exclusion or entry equilibria prevail in the case manufacturers are restricted to linear wholesale prices.

\section{$3 \quad$ Linear wholesale prices}

We start by analyzing the case with intense (but not perfect) retail competition, so that retailers are left with almost no profits when they compete head-to-head. We show that exclusion arises as the unique equilibrium in this case. This is true regardless of the fixed costs the entrant faces.

To show this more generally than just linear demands, we take "almost homogenous" to mean that as retailer differentiation disappears (which we denote by $\gamma \rightarrow 1$ ), retailer prices converge to those that would arise in the corresponding Bertrand homogenous goods competition game. This means if both retailers face the same wholesale price in equilibrium, then retailers will each choose a price that can be made arbitrarily close to this common wholesale price by choosing $\gamma$ sufficiently close to 1 . It also means that if retailer 1 faces a lower wholesale price than retailer 2 in equilibrium, then retailer 1 will optimally set a price that can be made arbitrarily close to retailer 2's higher wholesale price by choosing $\gamma$ sufficiently close to 1 . This will allow it to capture almost all (if not all) sales in the market. The linear demand model introduced in Section 2 satisfies these basic properties.

Proposition 1 Suppose that the incumbent can only make simultaneous and non-discriminatory exclusive offers to each retailer. If retailers are almost homogenous, then with linear wholesale pricing there is a unique exclusion equilibrium with the incumbent obtaining (almost) monopoly profits.

Proof. Consider what happens in each of the possible subgames following exclusive deals being signed (if any). To start with, suppose $S=2$, so both retailers accept $I$ 's exclusive contract. $I$ achieves 
monopoly profits in the limit by setting a common wholesale price $w$ that tends to the monopoly price $p_{M}$ from below. This induces retailers to set prices arbitrarily close to $w$ as $\gamma \rightarrow 1$. So $\pi_{i \mid S=2} \rightarrow 0$ and $\Pi_{I \mid S=2} \rightarrow\left(p_{M}-c_{I}\right) Q\left(p_{M}\right)$, where $Q$ denotes the market demand when both retailers charge $p_{M}$.

Suppose next $S=0$, so both retailers reject $I$ 's exclusive contract and $E$ enters. Competition between $I$ and $E$ leads to both free retailers to buy through $E$ at a common wholesale price of $c_{I}$. At this wholesale price, $\Pi_{E \mid S=0} \rightarrow\left(c_{I}-c_{E}\right) Q\left(c_{I}\right)$ while retailers do no make more than an arbitrarily small amount of profit. I obtains no profit.

Finally, suppose $S=1$, so (say) retailer 1 signs the contract and retailer 2 remains free. Assume that $F$ is low enough that $E$ can enter in this case. We know $E$ will sell through retailer 2 with a wholesale price $w_{2}$ satisfying $c_{E}<w_{2} \leq c_{I}$ (if $E$ were to set $w_{2}>c_{I}, I$ could profitably attract retailer 2 and $E$ will get no profit). Given $I$ sells through retailer 1 exclusively and $E$ sells through retailer 2 exclusively, head-to-head competition then occurs at the upstream level. $E$ sets $w_{2}$ just below retailer 1's conjectured price so that its retailer will undercut and take almost all the market (if not the entire market). It undercuts by the minimum amount necessary to induce this result. I can only respond by lowering its wholesale price to retailer $1, w_{1}$, down to $c_{I}$, below which it will make a loss. The resulting equilibrium must be characterized by $w_{1}$ arbitrarily close to (or equal to) $c_{I}$ with $w_{1} \geq c_{I}$ and $w_{2}$ arbitrarily close to $c_{I}$ (or equal to $c_{I}$ ) with $w_{2} \leq c_{I}$. (Any potential equilibrium with $w_{1}<c_{I}$ involves $I$ choosing a weakly dominated strategy, so we rule it out.) Retailer 2 (and therefore $E$ ) will capture almost all (if not all) of the demand at a retail price that is arbitrarily close to $c_{I}$. This implies no firm makes more than an arbitrarily small amount of profit with the exception of $E$, which makes a profit of $\Pi_{E \mid S=1} \rightarrow\left(c_{I}-c_{E}\right) Q\left(c_{I}\right)$.

Now consider the decision of retailers to sign exclusive deals in the initial stage. For $\gamma$ sufficiently close to one, $I$ will ensure both retailers sign by offering some small positive compensation $x$. With this offer, if a retailer thinks the other retailer will not sign and it signs, it gets no more than an arbitrarily small amount of profit but receives the compensation of $x$, while if it does not sign, it gets no more than an arbitrarily small amount of profit. Thus, the retailer is better off signing provided $\gamma$ is sufficiently close to one since any difference in retail profits can be made arbitrarily small. Alternatively, if the retailer thinks the other retailer will sign the exclusive deal in the initial stage, it is also better off signing since 
the payment of $x$ more than compensates for any loss in profits compared to deviating, which can be made arbitrarily close to zero for $\gamma$ sufficiently close to one (and is zero if $F$ is high such that $E$ does not enter in the $S=1$ subgame). For this reason, the compensation necessary to induce both firms to want to sign as the unique equilibrium can be made arbitrarily close to zero as $\gamma \rightarrow 1$. I's net profit $\Pi_{I \mid S=2}-2 x$ converges to the monopoly profit $\left(p_{M}-c_{I}\right) Q\left(p_{M}\right)$ as $\gamma \rightarrow 1$.

Proposition 1 shows that provided differentiation between downstream firms is sufficiently small, the unique equilibrium always involves retailers signing exclusive contracts that eliminate efficient entry, even if the incumbent can only offer simultaneous and non-discriminatory contracts and even if the rival manufacturer's cost of entry is arbitrarily small.

In the absence of exclusive deals, intense interbrand and intrabrand competition eliminates almost all the incumbent's and downstream competitors' profits. For this reason, the incumbent and downstream competitors always do better signing exclusive deals that lock out the entrant, thereby being able to share the monopoly profits of the industry. Given the incumbent gets to make the exclusive offer and given that retailers obtain almost no profit if they do not sign the offer, in equilibrium the incumbent retains almost all of the resulting profit. Even if a retailer deviates and does not sign, and the rival manufacturer enters, the retailer will obtain almost no profit given the entrant has no incentive to leave the deviating retailer with any profit once it has already entered.

This result highlights the key difference between our analysis and that in Fumagalli and Motta. In our model, the existence of the signed retailer provides a competitive constraint on the price the free retailer can set, meaning it makes almost no profit. This ensures a retailer does not want to deviate and reject an exclusive contract. Fumagalli and Motta remove this constraint by assuming retailers have to incur a fixed cost to stay active so that the signed retailer has no hope of making any profit competing against the more efficient entrant's retailer and therefore prefers to be inactive. With the signed retailer inactive, the remaining free retailer can then earn monopoly profits, ensuring any retailer has a strong incentive to reject an exclusive contract if they expect the other retailer to sign.

Corresponding to our setting, Fumagalli and Motta also discuss the case in which retailers do not face a fixed cost to be active. With identical retailers, linear wholesale pricing and linear demand, they note both entry and exclusion equilibria are possible. The entry equilibrium arises when the incumbent 
sets a high wholesale price to its signed buyer, given the signed buyer anyway attracts no demand in the $S=1$ subgame. The exclusion equilibrium arises, if as in Proposition 1, the incumbent sets a sufficiently competitive wholesale price to its signed buyer. Proposition 1 shows that allowing for a little bit of retailer differentiation provides a way of selecting between these multiplicity of equilibria, indicating that it is the exclusion equilibrium that remains. The proposition shows this conclusion does not depend on a particular specification of demand or the extent of the entrant's cost advantage. Even when the entrant's cost advantage is almost drastic, the same conclusion holds provided retailers are sufficiently strong competitors.

Once we allow for less intense competition between retailers, the analysis becomes more complicated (and interesting). One complication arises due to the possibility of a coordination failure between retailers. Since differentiated retailers bring with them some loyal customers, the entrant may only be able to cover its fixed cost if it can attract more than one retailer. ${ }^{3}$ This gives rise to a coordination problem, as in the existing naked exclusion literature, whereby if one retailer signs then the entrant will not enter, so that it is always an equilibrium for both retailers to sign. Rasmusen et al. (1991) and Segal and Whinston (2000) also find an equilibrium where entry is possible when the incumbent can only use simultaneous and non-discriminatory contracts. The next proposition shows this coordination problem disappears when downstream firms compete, provided they are not too differentiated. Sufficient competition between retailers removes the possibility of an equilibrium with entry, and instead a unique exclusion equilibrium arises in this case. We use linear demands to characterize this relationship precisely.

Proposition 2 Suppose that the incumbent can only make simultaneous and non-discriminatory exclusive offers to each retailer. If the fixed costs of entry are sufficiently high so that the entrant requires both retailers to profitably enter, then with linear wholesale pricing and linear demands there is a unique exclusion equilibrium if there is strong downstream competition $(\gamma \geq 1 / 2)$, while there is both an exclusion equilibrium and an entry equilibrium if there is weak downstream competition $(\gamma<1 / 2)$.

Proof. Consider what happens in each of the possible subgames following exclusive contracts being signed (if any).

\footnotetext{
${ }^{3}$ With almost homogenous retailers, it makes no difference to the entrant whether one or both retailers are available, which is why Proposition 1 does not depend on $F$.
} 
Suppose $S=2$, so both retailers accept $I$ 's exclusive offers. $E$ will not enter and $I$ will be a monopolist. $I$ chooses wholesale prices to each retailer, $w_{1}$ and $w_{2}$, to maximize $\left(w_{1}-c_{I}\right) q_{1}\left(p_{1}, p_{2}\right)+$ $\left(w_{2}-c_{I}\right) q_{2}\left(p_{2}, p_{1}\right)$, where $I$ takes into account the retailer pricing equilibrium in the final stage given the wholesale prices it sets. With linear demand, the retailers will be charged the monopoly wholesale price $w_{1}=w_{2}=\left(\alpha+c_{I}\right) / 2$, and equilibrium retail prices will be

$$
p_{1}=p_{2}=\frac{(3-2 \gamma) \alpha+c_{I}}{2(2-\gamma)}
$$

$I$ earns

$$
\Pi_{I \mid S=2}=\frac{\left(\alpha-c_{I}\right)^{2}}{2 \beta(1+\gamma)(2-\gamma)}
$$

and both retailers earn (before compensation)

$$
\pi_{i \mid S=2}=\frac{(1-\gamma)\left(\alpha-c_{I}\right)^{2}}{4 \beta(1+\gamma)(2-\gamma)^{2}}
$$

If instead $S=1$, so one retailer accepts $I$ 's exclusive deal, $E$ will not enter given fixed costs are assumed too high. The continuation equilibrium is identical to the case with $S=2$.

Now suppose $S=0$, so both retailers reject $I$ 's exclusive deal and $E$ enters. In this subgame, retailers will buy from the manufacturer which offers the lowest wholesale price. In equilibrium, $I$ offers $c_{I}$ to both retailers. This is the best $I$ can offer while covering its costs. $E$ will match this so as to attract both retailers. However, it has no reason to further lower wholesale prices given its profits are in fact increasing in $w_{E}$ up to $\left(\alpha+c_{E}\right) / 2>c_{I}$. Retailers then price at $p_{1}=p_{2}=\left((1-\gamma) \alpha+c_{I}\right) /(2-\gamma) . I$ earns zero profits while $E$ earns

$$
\Pi_{E \mid S=0}=\frac{2\left(c_{I}-c_{E}\right)\left(\alpha-c_{I}\right)}{\beta(1+\gamma)(2-\gamma)}
$$

and both retailers earn $\pi_{i \mid S=0}=4 \pi_{i \mid S=2}$.

Now consider the decision of retailers to sign exclusive deals. For any given $x>0$, there is always an equilibrium where both retailers sign. If one retailer deviates and does not sign, since $E$ will then not enter, such a retailer is worse off by $x$. (Given our tie-breaking rule that the retailers sign if they are indifferent, this set of equilibria extends to the case with $x=0$ ). On the other hand, there may or may not be an entry equilibrium. If neither retailer signs, then $E$ enters and retailers get $4 \pi_{i \mid S=2}$. If one retailer 
deviates and signs, since $E$ will then not enter, the retailer gets $\pi_{i \mid S=2}+x$ which is better than not signing if and only if $x \geq 3 \pi_{i \mid S=2}$. Thus, if $0 \leq x<3 \pi_{i \mid S=2}$ there is both an entry and an exclusion equilibrium, while if $x \geq 3 \pi_{i \mid S=2}$ there is only an exclusion equilibrium. The maximum amount of compensation $x$ the incumbent can offer is $\Pi_{I \mid S=2} / 2$, where $\Pi_{I \mid S=2} / 2>3 \pi_{i \mid S=2}$ if $\gamma>1 / 2, \Pi_{I \mid S=2} / 2=3 \pi_{i \mid S=2}$ if $\gamma=1 / 2$, and $\Pi_{I \mid S=2} / 2<3 \pi_{i \mid S=2}$ if $\gamma<1 / 2$. Therefore, if $\gamma \geq 1 / 2$ there is a unique exclusion equilibrium, while if $\gamma<1 / 2$ there is both an exclusion equilibrium and an entry equilibrium.

The proposition shows that the result in Proposition 1 extends to the case with moderate levels of retail competition provided the fixed costs of entry mean the rival manufacturer cannot recover its entry costs without attracting both retailers. Inefficient exclusion still prevails as the unique equilibrium in this case. However, the incumbent now has to share more of its monopoly profit with the retailers to achieve this outcome.

One way to contrast the implications of Proposition 2 with the existing literature on exclusive dealing, where buyers are final consumers, is to assume buyers can coordinate. If buyers can coordinate, exclusive deals can no longer be used to deter efficient entry in the naked exclusion framework of Rasmusen et al. (1991) and Segal and Whinston (2000). Rather, the Chicago School logic (Posner, 1976 and Bork, 1978) prevails. Buyers coordinate on the equilibrium with entry since interbrand competition raises the total surplus available to them. Proposition 2 says when buyers are retailers that compete downstream, the Chicago School defence of exclusive dealing need not apply. Rather, provided they are not too differentiated, retailers will coordinate on signing exclusive deals so that the industry remains monopolized, which ensures greater profits for themselves. While it is true only exclusive deals that increase the surplus to buyers will be signed, this does not mean exclusive deals will only be used when they enhance efficiency. Making buyers (retailers) better off now comes at the expense of final consumers. Welfare is lower both because of higher retail prices and because the entrant's more efficient production technology is not exploited. Thus, efficient entry can indeed be deterred by exclusive deals.

Exclusion eliminates one layer of competition - the competition between manufacturers. Whether this increases or decreases total profits available to retailers depends on the extent of downstream competition. If retailers face little competition, then eliminating competition between manufacturers leads to double marginalization and lower total profits. However, if retailers face strong competition then elim- 
inating competition between manufacturers results in industry profits that are closer to the monopoly level. Since the incumbent can share these high profits with the retailers through compensation offered in exclusive contracts, retailers will prefer exclusion whenever downstream competition is sufficiently strong. For the linear demand specification used, profit available to a retailer under exclusion $\left(\Pi_{I \mid S=2} / 2+\pi_{i \mid S=2}\right)$ is greater than under entry $\left(\pi_{i \mid S=0}\right)$ if and only if $\gamma>1 / 2$, which explains the specific result in Proposition $2 .^{4}$

Clearly, one reason why exclusion may not be possible is that with weak retailer competition, double marginalization losses offset any gains the retailers can obtain by restricting upstream competition. Section 4 explores the implications of allowing upstream firms to use two-part tariffs to eliminate double marginalization effects, showing this leads to an exclusion equilibrium even if downstream competition is weak. Another way to overcome retailers coordinating on the entry equilibrium when retail competition is weak is to use discriminatory or sequential deals. This eliminates the entry equilibrium, even for $\gamma<1 / 2$, and more generally reduces the amount of compensation required to exclude. For instance, with discriminatory contracts, it is sufficient for the incumbent to offer an attractive contract to a single retailer in order to eliminate the equilibrium in which both retailers do not sign (thereby excluding the entrant). This is always feasible since $\pi_{i \mid S=0}-\pi_{i \mid S=2} \leq \Pi_{I \mid S=2}$ for any $0 \leq \gamma<1$. This still requires the incumbent to offer a positive compensation, for instance, if the retailers coordinate on the equilibrium which maximizes their joint profits. The incumbent can exclude without any compensation once sequential contracts are allowed. Even if it receives no compensation, the first retailer anticipates that if it rejects the offer, the incumbent will still exclude by giving the second retailer a sufficient compensation. Given this, the first retailer will sign without compensation, and so will the second. As in the existing naked exclusion literature, sequential contracts work by dividing the two retailers, attracting one retailer to sign, and getting the second retailer "for free". We summarize this result here.

Proposition 3 Suppose that the incumbent can make sequential offers to the retailers. If the fixed costs of entry are sufficiently high so that the entrant requires both retailers to profitably enter, then with linear

\footnotetext{
${ }^{4}$ If instead demand is specified as $q_{i}=\alpha-\beta p_{i}+\gamma\left(p_{j}-p_{i}\right) /(1-\gamma)$ along the lines of Shubik and Levitan (1980), in which aggregate demand does not depend on the degree of substitution between retailers, the critical value of $\gamma$ in Proposition 2 becomes $\beta /(1+\beta)$ rather than $1 / 2$. When demand is less sensitive to price, double marginalization problems become less important and total profits are maximized under exclusion with a lower level of downstream competition.
} 
wholesale pricing and linear demands, there is a unique exclusion equilibrium regardless of the degree of downstream competition.

When the entrant's fixed costs are low, it may only need to attract a single retailer to make entry profitable. For instance, when retail competition is strong, the entrant can obtain almost the same profit from attracting just a single retailer as it obtains from attracting both retailers, given almost all consumers will then buy from the low cost retailer. Any retailer that does not sign a contract, will enable an entrant to enter, thereby shifting some profit from the rival retailer to itself. This might suggest that efficient entry cannot be prevented by exclusive contracts. With differentiated retailers, this impression is incorrect as Proposition 1 has already shown. However, if retailers are moderately or highly differentiated and the entrant only needs to attract one retailer to profitably enter, a deviating retailer may indeed obtain greater profits by buying from the entrant.

By deviating, the free retailer forces the homogenous manufacturers to compete to gain access to its customers. As a result it will enjoy a lower wholesale price compared to the signed retailer, and will obtain a greater share of the retail market. When competition in the retail market is sufficiently limited, this gain in retail profits will be more than the incumbent can afford to offer to eliminate upstream competition given the incumbent and retailers then face a double marginalization problem. An entry equilibrium can indeed prevail. In general, this happens when the profit the deviating retailer obtains under entry $\left(\pi_{i \mid S=1}\right)$ is greater than its share of monopoly profits under exclusion $\left(\Pi_{I \mid S=2} / 2+\pi_{i \mid S=2}\right)$. Working through the algebra for the linear demand case, this is true provided retailers are sufficiently differentiated, as the following proposition demonstrates.

Proposition 4 Suppose the fixed costs of entry are low enough so that the entrant can enter even if just one retailer is available, that upstream firms are restricted to linear wholesale prices and final demand is linear. Provided downstream competition is not too strong $(\gamma<0.76)$, then we have a unique entry equilibrium. Provided downstream competition is sufficiently strong $(\gamma>0.94)$, then we have a unique exclusion equilibrium. For an intermediate level of downstream competition, we have a unique exclusion equilibrium provided the entrant's cost advantage is not too strong $(\theta<0.60$ is sufficient) and a unique entry equilibrium provided the entrant's cost advantage is sufficiently strong $(\theta>0.65$ is sufficient), with the critical value of $\theta$ decreasing in $\gamma$ for intermediate cases. Figure 1 illustrates. 
Proof. The proof is lengthy as it requires three different regions of $\gamma$ to be analyzed separately. It is relegated to the appendix.

\section{- - - Figure 1 here - - -}

Combined, Propositions 2 and 4 give results for the full range of downstream competition, fixed costs of entry and cost advantage of the entrant in the linear demand case. Whether an exclusion or entry equilibrium prevails depends on both the extent of downstream competition, the level of fixed costs and the extent of the entrant's cost advantage. This relationship is illustrated by way of two figures. Figure 1 does this for the case in which fixed costs are low enough that the entrant only needs one retailer to profitably enter (Proposition 4). It shows how the nature of equilibrium depends on the degree of downstream competition $\gamma$ and the entrant's cost advantage $\theta$. The figure highlights that for a range of intermediate levels of downstream competition, whether we have exclusion or entry depends on the extent of the entrant's cost advantage.

When the fixed costs of entry are higher, Proposition 2 applies and the cutoff between exclusion and entry is independent of the cost advantage of the entrant. Thus, whether the fixed costs of entry are high or low also has an important bearing on the nature of equilibria. Figure 2 illustrates this by showing how the nature of equilibria vary for the full range of $F$ and $\gamma$, holding constant the entrant's cost advantage by setting $\theta$ equal to $1 / 2$, which means the incumbent's marginal cost is halfway between the entrant's marginal cost and the entrant's monopoly price. The figure applies for any valid value of $\alpha, \beta$ and $c_{E}$ since these simply scale the values presented. ${ }^{5}$ The two "horizontal" curves in each figure represent the critical values of $F$ that determine whether the rival manufacturer can profitably enter with one or two retailers. The upper curve is defined by $F=\Pi_{E \mid S=0}$ and the lower curve is defined by $F=\Pi_{E \mid S=1}$. The vertical lines in each figure indicate critical values of $\gamma$ that determine the type of equilibria that exist.

The three different expressions for $\Pi_{E \mid S=1}$ in the proof of Proposition 4 determine the critical values of $F$, below which entry is feasible even if just one retailer is available (the condition for Proposition 4 to apply) and above which entry requires both retailers to be available (the condition for Proposition 2 to apply). This explains the kinks in the $F=\Pi_{E \mid S=1}$ curve in figure 2 .

\footnotetext{
${ }^{5}$ The value of $F$ is scaled by dividing by $\left(\alpha-c_{E}\right)^{2} / \beta$ so it lies exactly between 0 and 1.
} 
When the fixed costs of entry are too high (above the upper curve), entry is never possible and so the incumbent maintains its monopoly regardless of the use of exclusive deals or not. In this paper, we have focused only on fixed costs for which entry is viable. For fixed costs of entry in which the entrant requires both retailers to profitably enter, we get the intermediate region between the two curves. If $\gamma<1 / 2$ we get either entry or exclusion, while with $\gamma \geq 1 / 2$ exclusion is unique. This corresponds to the results in Proposition 2, in which the incumbent can only make simultaneous and non-discriminatory offers to each retailer. When the incumbent can offer discriminatory or sequential contracts, these entry equilibria are eliminated (this is the only effect of allowing discriminatory or sequential contracts on the figures). The remaining region, below the $F=\Pi_{E \mid S=1}$ curve, is where fixed costs of entry are sufficiently low that the entrant only needs one retailer to be able to profitably enter. Note this region expands rapidly as $\gamma$ approaches 1 , reflecting the fact that the profits from attracting one or two retailers are almost identical when the retailers are almost homogenous. Again, the region can be divided into two cases depending on the value of $\gamma$, corresponding to Proposition 4. Regardless of the value of $F$, for sufficiently strong intrabrand competition $(\gamma>0.76)$, the outcome is always exclusion. ${ }^{6}$

Figures 1 and 2 reveal the overall tendency for exclusion to be more likely when competition between downstream firms is strong. This is the opposite conclusion to that found in Fumagalli and Motta, who argue that downstream competition makes anticompetitive exclusion less likely to be sustainable. Thus, contrary to them, we predict exclusive dealing should be more prevalent in industries with highly competitive downstream markets than those which are strongly differentiated. However, figures 1 and 2 reveal this prediction does not always hold.

Figure 1 shows that there is a small range of the entrant's cost advantage ( $\theta$ between 0.60 and 0.65$)$ for which as retailers become more homogenous we go from an entry equilibrium to exclusion, back to entry again, and finally to an exclusion equilibrium. The first comparative static, from entry to exclusion equilibrium as retailers become more homogenous is standard. However, the change back to an entry equilibrium reflects that when the entrant has a sufficient cost advantage, an increase in downstream

\footnotetext{
${ }^{6}$ As shown in figure 1 , when the entrant has a large cost advantage, stronger intrabrand competition is required for exclusion to arise, with the result always holding provided $\gamma>0.94$.
} 
competition can cause the entrant to lower its wholesale price below that offered by the incumbent so that the free retailer takes a much larger share of the market. This generates greater profit for the free retailer, explaining why the incumbent will not be able to exclude. However, as retail competition increases further, the entrant no longer has to lower its wholesale price by much, so that eventually the free retailer's profit (even when it monopolizes the market) becomes less than what it can achieve signing with the incumbent. Exclusion is eventually restored.

Similarly, as is clear from figure 2, for a small range of fixed costs of entry there is also a nonmonotonic relationship between the nature of equilibria and the degree of product differentiation. This reflects that increasing downstream competition has two effects. First, it reduces the benefit to retailers from having upstream competition making exclusion more likely. Second, it makes it easier for an entrant to achieve scale economies by attracting a single buyer, thereby making entry easier. The second effect only matters when fixed costs are close to the critical level at which the entrant would (just) need both retailers to profitably enter, explaining why the non-monotonic effect only arises for a small range of fixed costs. Within this range, an increase in the degree of downstream competition can first make exclusion easier, as the first effect dominates. Then as downstream competition intensifies further, the second effect dominates, making entry easier. Finally, with intense downstream competition, the second effect becomes redundant since exclusion arises regardless of how many retailers the entrant needs to attract to profitably enter.

Figures 1 and 2 can also be used to compare our results with the related literature on naked exclusion. The seminal works of Rasmusen et al. (1991) and Segal and Whinston (2000) assume buyers are final consumers. They focus on the case in which there is a coordination failure between buyers. In figure 2, this is equivalent to just the region between $F=\Pi_{E \mid S=1}$ and $F=\Pi_{E \mid S=0}$ with $\gamma$ set to zero. They find both exclusion and entry equilibria are possible (as in figure 2), but the entry equilibrium can be eliminated with the use of discriminatory or sequential contracts.

Fumagalli and Motta extend these earlier models to consider the extreme case of homogenous Bertrand competition, considering the same range of fixed costs. In the present setting (where both retailers remain active), this would give rise to multiple entry and exclusion equilibria, even when the incumbent can use sequential contracts. An exclusion equilibrium is possible if in the case of only one retailer signing, 
the incumbent charges the signed retailer a wholesale price close to its marginal cost, while an entry equilibrium is possible if the incumbent charges the signed retailer a high enough wholesale price. The incumbent is indifferent between these different possibilities since the signed retailer anyway obtains no demand. However, the deviating retailer is not indifferent, since its profit depends on the wholesale price that the signed buyer faces. Allowing for a small amount of differentiation between retailers provides one way to eliminate this multiplicity of equilibria. Our results show that it is the exclusion equilibrium that then prevails.

In a separate appendix, Fumagalli and Motta also consider the case in which retailers are homogenous Cournot competitors. In terms of the strength of downstream competition, quantity competition is equivalent to price competition when retailers are moderately differentiated. Thus, their finding of entry with low fixed costs of entry and exclusion with high fixed costs of entry, is just a special case of the more general result illustrated in figure 2 .

It is more difficult to relate our findings to those of Simpson and Wickelgren (2004), given their different cost specification (ex-ante, the incumbent and entrant face symmetric costs) and that they allow buyers to renegotiate exclusive contracts. Their finding that the threshold level of downstream competition necessary for the incumbent to use exclusive deals to deter entry does not depend on entrant's minimum efficient scale is equivalent to the vertical line $\gamma=0.76$ in Figure 2. However, figures 1 and 2 reveal that under our cost specification, this independence is not general. Figure 1 shows when the entrant has a strong cost advantage $(\theta>0.65)$, the threshold is higher $(\gamma=0.94)$. For higher levels of fixed cost, so that both retailers are required to profitably enter, the threshold level is substantially lower $(\gamma=0.50)$. Moreover, if upstream firms sell to downstream firms using two-part tariffs, the threshold level of $\gamma$ shifts all the way back to zero, since exclusion arises as an equilibrium for any $0<\gamma<1$. We turn to this case next.

\section{Two-part tariffs}

In this section, we extend our previous analysis to allow upstream firms to compete in simple two-part tariffs in stage 3 . These consist of a linear wholesale price and an upfront fixed fee, denoted $(w, \phi)$. These can be motivated by assuming a manufacturer cannot observe retailers' prices, sales or profits, and nor 
can they observe how much retailers buy from the rival manufacturer or the details of its contract offer. In particular, these simple two-part tariffs rule out deviations in which an upstream firm offers a high wholesale price and a subsidy (negative $\phi$ ) since a retailer will then accept the offer to receive the subsidy but purchase from the lower priced manufacturer.

The main impact of upstream firms using two-part tariffs is to remove the double marginalization that arises under linear wholesale pricing when using exclusion to restrict upstream competition given that retailers are differentiated. We thus find that allowing upstream firms to use two-part tariffs, exclusion arises regardless of the strength of downstream competition. Moreover, we do not need to assume demand is linear to obtain the result. Rather, we just posit general differentiated Bertrand retailers, where demand satisfies the usual properties (Singh and Vives, 1984, Section 5) so that optimizing prices uniquely exist for each retailer and prices are strategic complements.

Proposition 5 Suppose that the incumbent can only make simultaneous and non-discriminatory exclusive offers to each retailer. If upstream firms can offer two-part tariffs, then there is a unique exclusion equilibrium regardless of the degree of downstream competition.

Proof. We suppose that $E$ 's fixed cost is sufficiently small that in the $S=1$ subgame, $E$ is capable of entering. (If fixed costs are higher so $E$ needs both retailers to profitably enter, the existence of an exclusion equilibrium becomes trivial since there is no advantage to a retailer of rejecting the contract when the other retailer is expected to sign. The uniqueness of this equilibrium follows from the same argument as used below).

Suppose first $S=2$, so both retailers accept $I$ 's exclusive offers. $E$ will not enter and $I$ will be a monopolist selling to the two signed retailers. I maximizes its profit by setting its wholesale prices so that the competing retailers set their retail prices at the monopoly level $p_{M}$ in equilibrium. It extracts the retailers' profits through franchise fees. I thus obtains the monopoly profit of the industry

$$
\Pi_{I \mid S=2}=2\left(p_{M}-c_{I}\right) q\left(p_{M}, p_{M}\right)
$$

while retailers obtain nothing. ${ }^{7}$

\footnotetext{
${ }^{7}$ This outcome assumes $I$ can overcome the commitment problem of Hart and Tirole (1990), which arises here because I's offers are assumed unobservable. This problem is ruled out with our assumption on beliefs, that if a retailer receives a lower wholesale price from $I$, it assumes $I$ also makes the same offer to the rival retailer (see Rey and Vergé, 2004).
} 
If instead $S=1$, so one retailer accepts $I$ 's exclusive deal and the other retailer remains free, the upstream firms compete for the free retailer. Let $I$ 's offers be $\left(w_{S}, \phi_{S}\right)$ to the signed retailer and $\left(w_{I}, \phi_{I}\right)$ to the free retailer, and let $E$ 's offer be $\left(w_{E}, \phi_{E}\right)$ to the free retailer. $I$ will price down to cost $\left(w_{I}=c_{I}\right.$ and $\left.\phi_{I}=0\right)$ in an attempt to attract the free retailer. Given E's cost advantage, it will always attract the free retailer in equilibrium. ${ }^{8}$

Given it uses its fixed fee to extract all of the signed retailer's profit, $I$ chooses $w_{S}$ to maximize its profit of $\left(p\left(w_{S}, p_{2}\right)-c_{I}\right) q\left(p\left(w_{S}, p_{2}\right), p_{2}\right)$, where $p\left(w_{S}, p_{2}\right)$ is chosen by the signed retailer to maximize $\left(p-w_{S}\right) q\left(p, p_{2}\right) . I$ can make sure the retailer's maximization problem coincides with its own maximization problem by setting $w_{S}=c_{I}$, which is therefore the profit maximizing wholesale price. The free retailer's alternative to $E$ 's offer is to buy from $I$ at $c_{I}$. This gives it the standard duopoly retail profit corresponding to each retailer buying at $c_{I}$, which we write as

$$
\pi_{D}=\left(p_{D}-c_{I}\right) q\left(p_{D}, p_{D}\right)
$$

where $p_{D}$ is the normal duopoly price when upstream firms compete directly and have costs of $c_{I}$. To attract the free retailer, $E$ will therefore offer the free retailer exactly $\pi_{D}$. Since $\pi_{D}<\Pi_{I \mid S=2} / 2, I$ can always offer sufficient compensation $x$ as part of its exclusive deals such that neither firm will want to deviate and become the free retailer when the other signs the exclusive deal. In other words, an exclusion equilibrium always exists.

Now consider the subgame with $S=0$ so both upstream firms compete for both retailers. Given it is more efficient, $E$ will attract both retailers in equilibrium. $I$ offers its best terms $\left(c_{I}, 0\right)$ to both retailers. $E$ only has to match this to attract both retailers and earn a profit of $2\left(c_{I}-c_{E}\right) q\left(p\left(c_{I}, c_{I}\right), p\left(c_{I}, c_{I}\right)\right) .{ }^{9}$ The corresponding retail profit is the normal duopoly profit $\pi_{D}$ characterized above. If it has a sufficient

\footnotetext{
${ }^{8}$ Deviations in which $I$ attracts the free retailer with a higher wholesale price and a bribe are ruled out since with a simple two-part tariff involving a bribe, the free retailer will accept the offer to receive the bribe but still buy from $E$ at the lower wholesale price.

${ }^{9}$ A similar offer by $E$ undermines the equilibrium proposed in Proposition 2 of Fumagalli and Motta with homogenous retailers, which they claim involves one retailer (retailer 2) buying from $E$ at $w_{E}=c_{E}$ and $\phi_{E}=\pi_{M}\left(c_{E}\right)-\pi_{M}\left(c_{I}\right)$, with $I$ offering $\left(c_{I}, 0\right)$ but obtaining no profit since retailer 1 is inactive. Even within their own setting (of public offers and small fixed costs of retailers being active), their proposed equilibrium affords a profitable deviation. Specifically, $E$ does better setting $w_{E}=c_{I}$ and (if necessary) setting $\phi_{E}$ slightly negative to both retailers. Regardless of what the other retailer does, from the perspective of each retailer, this contract always dominates $I$ 's offer of $\left(c_{I}, 0\right)$ or being inactive. With both retailers
} 
cost advantage, $E$ may be able to do better offering $\left(w_{E}, \phi_{E}\right)$ to both retailers, with $w_{E}<c_{I}$ and $\phi_{E}>0$. This allows $E$ to extract greater profit from retailers through its franchise fee since it makes it less profitable for a single retailer to deviate and buy from $I$ given it will then be competing against a lower cost retailer. Thus, the equilibrium will involve $I$ offering $\left(c_{I}, 0\right)$ and $E$ offering $\left(w_{E}, \phi_{E}\right)$ with $w_{E} \leq c_{I}$ and $\phi_{E} \geq 0$, with retailers left with at most $\pi_{D}$, and possibly less than this. ${ }^{10}$ Since $\pi_{D}<\Pi_{I \mid S=2} / 2, I$ can always offer sufficient compensation $x$ as part of its exclusive deals such that a firm will want to sign even if the other does not, meaning there is no equilibrium where both retailers reject I's offer. That is, there is no entry equilibrium and therefore the only equilibrium involves exclusion.

Proposition 4 established that when upstream firms are restricted to competing in linear wholesale prices, there is a unique entry equilibrium when downstream competition is sufficiently weak and scale economies are not too great. The above proposition shows that this entry equilibrium is eliminated once upstream firms can make use of two-part tariffs, with exclusion arising instead as the unique outcome. With two-part tariffs, the double marginalization problem which limits monopoly profits under exclusion no longer applies. Thus, the incumbent can potentially offer the industry monopoly profits to get retailers to sign. Moreover, a retailer that deviates and does not sign with the incumbent when the other does, will at most be able to extract its normal duopoly profit in which both retailers buy at the incumbent's $\operatorname{cost} c_{I}$. The entrant has no reason to leave the free retailer with more profit given the incumbent is only willing to price down to $c_{I}$ to attract the free retailer. Since duopoly profit is less than monopoly profit, retailers can always be made better off signing the exclusive contracts.

accepting, homogenous Bertrand competition will cause retail prices to be set at $c_{I}$, which gives $E$ a profit of approximately $\left(c_{I}-c_{E}\right) Q\left(c_{I}\right)$. This is greater than E's proposed equilibrium profit of $\pi_{M}\left(c_{E}\right)-\pi_{M}\left(c_{I}\right)$ in their linear demand setting. ${ }^{10}$ One can also rule out a deviation by $I$ involving it setting $w_{I}<c_{I}$ and $\phi_{I}>0$. Even if $w_{E}=c_{I}$, this is not profitable. Our assumption on beliefs ensures each retailer assumes the other retailer receives the same offer. This prevents retailers being fooled by such a deviation, which otherwise could afford $I$ a profitable deviation. Formally, $I$ needs to set $\phi_{I}<\left(p^{*}-w^{*}\right) q\left(p^{*}, p^{*}\right)-\pi_{D}$, where $w^{*}<c_{I}$ and $p^{*}=\arg \max _{p}\left(p-w^{*}\right) q\left(p, p^{*}\right)$, to both retailers in order for both of them to deviate. This deviation is only profitable for $I$ if $\phi_{I}>\left(c_{I}-w^{*}\right) q\left(p^{*}, p^{*}\right)$. Combining these two incentive constraints implies $\pi_{D}<\left(p^{*}-c_{I}\right) q\left(p^{*}, p^{*}\right)$ which clearly is not true since $p^{*}<p_{D}$ and $p_{D}$ is below the monopoly price. 


\section{Conclusion}

The ability of an established firm to use exclusive deals to foreclose a market when buyers compete downstream has recently been examined (Fumagalli and Motta, 2006 and Simpson and Wickelgren, 2004). This paper adds to the study of this important case by analyzing a standard model of naked exclusion allowing for downstream firms to engage in imperfect competition. Unlike the existing literature, we consider the full range of possible degrees of downstream competition, fixed costs of entry, and cost efficiencies of the entrant, as well as analyzing both the case where upstream firms are restricted to offering linear wholesale prices and where they can offer two-part tariffs.

The main result we obtain is that inefficient exclusion prevails when a downstream firm's share of monopoly profits under exclusion exceeds the profit it can obtain with upstream entry when others buy from the less efficient incumbent. The degree of downstream competition has three effects on this comparison. Strong downstream competition means a deviating buyer that buys from the entrant at a lower cost can induce entry even if all other buyers sign with the incumbent since it can monopolize the retail market. This undermines the coordination failure argument for exclusion of Rasmusen et al. (1991) and Segal and Whinston (2000), as Fumagalli and Motta point out. However, strong downstream competition also means that although the deviating buyer monopolizes the retail market in equilibrium, it obtains little profit from doing so. Finally, in the case of linear wholesale pricing, strong downstream competition means there is little double marginalization distortion associated with the upstream market being monopolized. Together, these effects mean that with linear wholesale pricing, inefficient exclusion prevails when downstream markets are sufficiently competitive but not when downstream firms are strongly differentiated. We show this is true regardless of the fixed costs of entry, or the extent to which the entrant enjoys (non-drastic) cost advantages. On the other hand, when upstream firms can compete in two-part tariffs to sell to downstream firms, exclusion prevails as an equilibrium regardless of the extent of downstream competition.

In this paper, exclusive deals are used to transfer surplus from final consumers to the established upstream and downstream firms by eliminating competition from a rival upstream entrant, thereby raising consumer prices. Such contracts may continue to be used even if they lower the total profits obtained by the downstream firms, but only when the incumbent can exploit a coordination failure between 
downstream firms. Ignoring any such coordination problem between downstream firms, our analysis is consistent with the logic of Posner (1976) and Bork (1978) that only exclusive deals that increase the surplus available to buyers will be signed. The difference between the earlier Chicago literature and the present result is that making buyers (downstream firms) better off through the use of exclusive contracts come at the expense of final consumers. Welfare is lower both because of higher retail prices and because the entrant's more efficient production technology is not exploited.

One natural extension of the current paper is to analyze what happens when we move from duopoly to oligopoly in the downstream market. On the one hand, oligopoly should make entry easier, since the monopoly profits from exclusion now has to be shared amongst a larger number of downstream firms. On the other hand, more downstream firms implies stronger downstream competition, and as this papers shows, this can help support exclusion.

A more fundamental extension is to allow the entrant to also offer exclusive deals. Even if the entrant is more efficient, this need not undermine exclusion. If the entrant can offer exclusive deals to buyers who remain unsigned before deciding whether to enter, a single buyer that signs with the entrant rather than the incumbent can be offered at most the entrant's duopoly profits less its fixed cost of entry. This could well be less than the buyer's share of monopoly profits from signing with the incumbent. Thus, even though an entrant needs only one buyer to profitably enter, a coordination failure between buyers may still arise, in which buyers would be jointly better off signing with the entrant, but both sign with the incumbent since they expect the other to do the same. Such expectations seem plausible if the incumbent has historically signed up the buyers.

Another future direction is to explore the implications of imperfect competition at the upstream level, so that the differentiation of the entrant's product rather than a cost advantage is its motive for entry. This is taken up by Wright (2006) in the case of intense downstream competition. If upstream competition is strong, then the mechanism is the same as in this paper. On the other hand, if upstream competition is weak, the incumbent does better leaving one downstream competitor free, so as to soften (rather than eliminate) competition by depriving the entrant of a competitive downstream market for its product. With differentiation at both levels, this opens up an efficiency reason for downstream firms to supply both upstream firms' products, perhaps making anticompetitive exclusive dealing less likely. In 
our view, this is the most interesting environment in which to study exclusive dealing, although also the most challenging.

\section{Appendix: Proof of Proposition 4}

Proof. The analysis for the $S=0$ and $S=2$ subgames is identical to that in the proof of proposition 2. However, the $S=1$ subgame differs because entry is now possible given the assumption on fixed costs in the proposition. In this subgame, the best stage 3 offer that $I$ can give the free retailer is $c_{I}$. $E$ will always at least match this to attract the free retailer. The question is, in competition with $I$ 's signed retailer (retailer 1), will $E$ want to set a lower wholesale price to allow retailer 2 to take a greater share of the market? There are three different regions of $\gamma$ for which prices and profits are determined differently. These reflect whether I's signed retailer is monopolized by the free retailer or whether the two retailers share the market, and in the latter case, whether the constraint on E's wholesale price caused by competition with $I$ for the free retailer is binding or not.

Let $\gamma_{L}$ and $\gamma_{H}$ be defined such that for $\gamma<\gamma_{L}, E$ sets its wholesale price offer to the free retailer at $c_{I}$ and for $\gamma>\gamma_{H}, E$ induces its retailer to monopolize the downstream market. Specifically, to derive these critical values of $\gamma$, we need to first derive the unique intersection of best response functions from the respective optimization problems of $I$ and $E$ :

$$
\begin{aligned}
& \max _{w_{1}}\left(w_{1}-c_{I}\right) q_{1}\left(p_{1}\left(w_{1}, p_{2}\right), p_{2}\right) \\
& \max _{w_{2}}\left(w_{2}-c_{E}\right) q_{2}\left(p_{2}\left(w_{2}, p_{1}\right), p_{1}\right) .
\end{aligned}
$$

The simultaneous solution to the above problem is given by the pair $\left(w_{1}^{*}, w_{2}^{*}\right)$ which denotes the optimal wholesale prices assuming no constraints are active. The critical value $\gamma_{L}$ is derived by solving $w_{2}^{*}=c_{I}$ for $\gamma$, which implies

$$
\gamma_{L}=\frac{\theta+\sqrt{196-340 \theta+145 \theta^{2}}-2}{3(4-3 \theta)},
$$

where we have used the definition that $c_{I}=\theta\left(\alpha+c_{E}\right) / 2+(1-\theta) c_{E}$. For lower levels of downstream competition, $E$ would like to set a higher wholesale price if not for the constraint imposed by competition with $I$. Therefore, for $\gamma \leq \gamma_{L}, E$ sets its wholesale price at $c_{I}$ to the free retailer in equilibrium. 
The critical value $\gamma_{H}$ is derived by solving $w_{1}^{*}=c_{I}$ for $\gamma$, which implies

$$
\gamma_{H}=\frac{\sqrt{49-48 \theta+12 \theta^{2}}-1}{3(2-\theta)} .
$$

Monopolization starts at the value of $\gamma$ such that even if $I$ charges $c_{I}$ and its signed retailer follows suit, then if $E$ sets its wholesale price according to market sharing, the free retailer is indifferent between setting a retail price that monopolizes the downstream market and setting a wholesale price that implies market sharing. For higher values of $\gamma$, the free retailer will strictly prefer monopolization. For lower values of $\gamma, I$ can still supply some demand at a wholesale price above cost.

Note that $\gamma_{L}<\gamma_{H}$ with both critical values decreasing in $\theta$. Moreover, $\gamma_{L} \in[0,1]$ and $\gamma_{H} \in$ $[(\sqrt{13}-1) / 3,1]$ for $\theta \in[0,1]$. Define three cases as follows: (i) $\gamma<\gamma_{L}$, (ii) $\gamma_{L} \leq \gamma \leq \gamma_{H}$ and (iii) $\gamma>\gamma_{H}$

Consider case (i). As noted above, in equilibrium $w_{2}=c_{I}$. I will therefore set $w_{1}$ to maximize $\left(w_{1}-c_{I}\right) q_{1}\left(p_{1}\left(w_{1}, p_{2}\right), p_{2}\right)$, retailer 1 will set $p_{1}$ to maximize $\left(p_{1}-w_{1}\right) q_{1}\left(p_{1}, p_{2}\right)$ and retailer 2 will set $p_{2}$ to maximize $\left(p_{2}-c_{I}\right) q_{2}\left(p_{2}, p_{1}\right)$. Given unobservable offers, these three maximization problems are solved simultaneously. In the resulting equilibrium,

$$
\Pi_{I \mid S=1}^{(i)}=\frac{2(1-\gamma)(2+\gamma)^{2}\left(\alpha-c_{I}\right)^{2}}{\beta(1+\gamma)\left(8-3 \gamma^{2}\right)^{2}}
$$

and

$$
\Pi_{E \mid S=1}^{(i)}=\frac{(4+3 \gamma)\left(\alpha-c_{I}\right)\left(c_{I}-c_{E}\right)}{\beta(1+\gamma)\left(8-3 \gamma^{2}\right)}
$$

The signed retailer earns (before compensation) $\pi_{1 \mid S=1}^{(i)}=\Pi_{I \mid S=1}^{(i)} / 2$ while the free retailer earns

$$
\pi_{2 \mid S=1}^{(i)}=\frac{(1-\gamma)(4+3 \gamma)^{2}\left(\alpha-c_{I}\right)^{2}}{\beta(1+\gamma)\left(8-3 \gamma^{2}\right)^{2}} .
$$

Consider case (ii). We still have sharing as before but E's wholesale price is no longer constrained by $c_{I}$ - it is set to maximize $\left(w_{2}-c_{E}\right) q_{2}\left(p_{2}\left(w_{2}, p_{1}\right), p_{1}\right)$. Equilibrium wholesale prices are determined by the simultaneous solution to this maximization problem plus the three maximization problems above. In the resulting equilibrium,

$$
\Pi_{I \mid S=1}^{(i i)}=\frac{2\left(\left(4-3 \gamma^{2}\right)\left(\alpha-c_{I}\right)-\gamma\left(\alpha-c_{E}\right)\right)^{2}}{\beta\left(1-\gamma^{2}\right)\left(16-9 \gamma^{2}\right)^{2}}
$$


and

$$
\Pi_{E \mid S=1}^{(i i)}=\frac{2\left(\left(4-3 \gamma^{2}\right)\left(\alpha-c_{E}\right)-\gamma\left(\alpha-c_{I}\right)\right)^{2}}{\beta\left(1-\gamma^{2}\right)\left(16-9 \gamma^{2}\right)^{2}}
$$

The signed retailer earns (before compensation) $\pi_{1 \mid S=1}^{(i i)}=\Pi_{I \mid S=1}^{(i i)} / 2$ while the free retailer earns $\pi_{2 \mid S=1}^{(i i)}=$ $\Pi_{E \mid S=1}^{(i i)} / 2$.

Consider case (iii). For this case, $E$ induces the free retailer to monopolize the downstream market. Given that the signed retailer sets its price equal to its cost $w_{1}, E$ sets the highest wholesale price such that the free retailer has an incentive to monopolize the downstream market. Under a monopolization equilibrium, we know that $w_{1}=c_{I}$ and thus the monopolization wholesale price is $w_{2}=\left(\left(2-\gamma^{2}\right) c_{I}-\alpha(1-\gamma)(2+\gamma)\right) / \gamma$. This wholesale price induces the free retailer to set its retail price equal to $\left(c_{I}-\alpha(1-\gamma)\right) / \gamma$ which is sufficient for monopolization. Both $I$ and the signed retailer earn zero profits. $E$ earns

$$
\Pi_{E \mid S=1}^{(i i i)}=\frac{\left(\alpha-c_{I}\right)\left(\gamma\left(\alpha-c_{E}\right)-\left(2-\gamma^{2}\right)\left(\alpha-c_{I}\right)\right)}{\beta \gamma^{2}}
$$

while the free retailer earns

$$
\pi_{2 \mid S=1}^{(i i i)}=\frac{\left(1-\gamma^{2}\right)\left(\alpha-c_{I}\right)^{2}}{\beta \gamma^{2}} .
$$

Now consider the retailers' signing decisions. For $k=i, i i, i i i$, an exclusion equilibrium exists if $\pi_{i \mid S=2}+\Pi_{I \mid S=2} / 2 \geq \pi_{2 \mid S=1}^{(k)}$ since then a retailer does worse rejecting an exclusive contract when the other retailer signs, while an entry equilibrium arises if $\pi_{i \mid S=0}>\pi_{1 \mid S=1}^{(k)}+\Pi_{I \mid S=2} / 2$ since then a retailer does worse signing a contract when the other does not. Substituting in the above profit expressions (and those from the proof of Proposition 2), and for each value of $\gamma$ determining the range of $\theta$ for which each equilibrium exists, gives a complete picture of the implied equilibria as a function of $0 \leq \gamma<1$ and $0<\theta<1$. This is shown in figure 1 in the text, which confirms the statement of the proposition. (In the statement of the proposition, we have rounded critical parameter values to two decimal places.)

\section{$7 \quad$ References}

Bernheim, B. D. and M. D. Whinston (1998) "Exclusive dealing," The Journal of Political Economy, 106(1): 64-103. 
Bowley, A. L. (1924) The Mathematic Groundwork of Economics. Oxford: Oxford University Press.

Bork, R. (1978) The Antitrust Paradox: A Policy at War with Itself. New York: Basic Books.

Fumagalli, C. and M. Motta (2006) "Exclusive dealing and entry, when buyers compete," American Economic Review, 96: 785-795

Hart, O. and J. Tirole (1990) "Vertical integration and market foreclosure," Brookings Papers on Economic Activity: Microeconomics: 205-286.

Katz, M. (1991) "Game-playing agents: Unobservable contracts as precommitments," RAND Journal of Economics, 22: 307-328.

Posner, R. A. (1976) Antitrust Law: An Economic Perspective. Chicago: University of Chicago Press.

Rasmusen E. B., J. M. Ramseyer, and J. J. S. Wiley (1991) "Naked exclusion," American Economic Review, 81: 1137-45.

Rey, P. and T. Vergé (2004) "Bilateral control with vertical contracts," RAND Journal of Economics, 35: $728-746$.

Segal, I. and M. D. Whinston (2000) "Naked exclusion: Comment," American Economic Review, 90: 296-309.

Shubik, M. and R. Levitan (1980) Market Structure and Behavior. Cambridge, MA: Harvard University Press.

Simpson, J. and A. L. Wickelgren (2004) "Naked exclusion, efficient breach, and downstream competition," mimeo.

Singh, N. and X. Vives (1984) "Price and quantity competition in a differentiated duopoly," RAND Journal of Economics, 15: 546-554.

Wright, J. (2006) "Naked exclusion and the anticompetitive accommodation of entry," mimeo, National University of Singapore. 


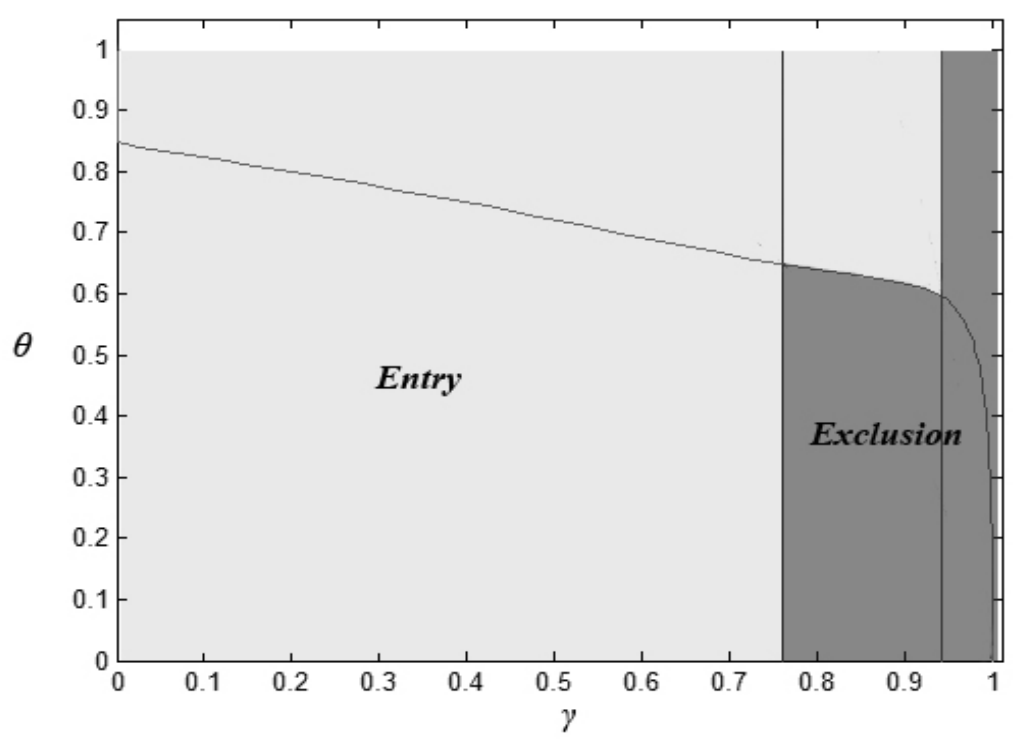

Figure 1

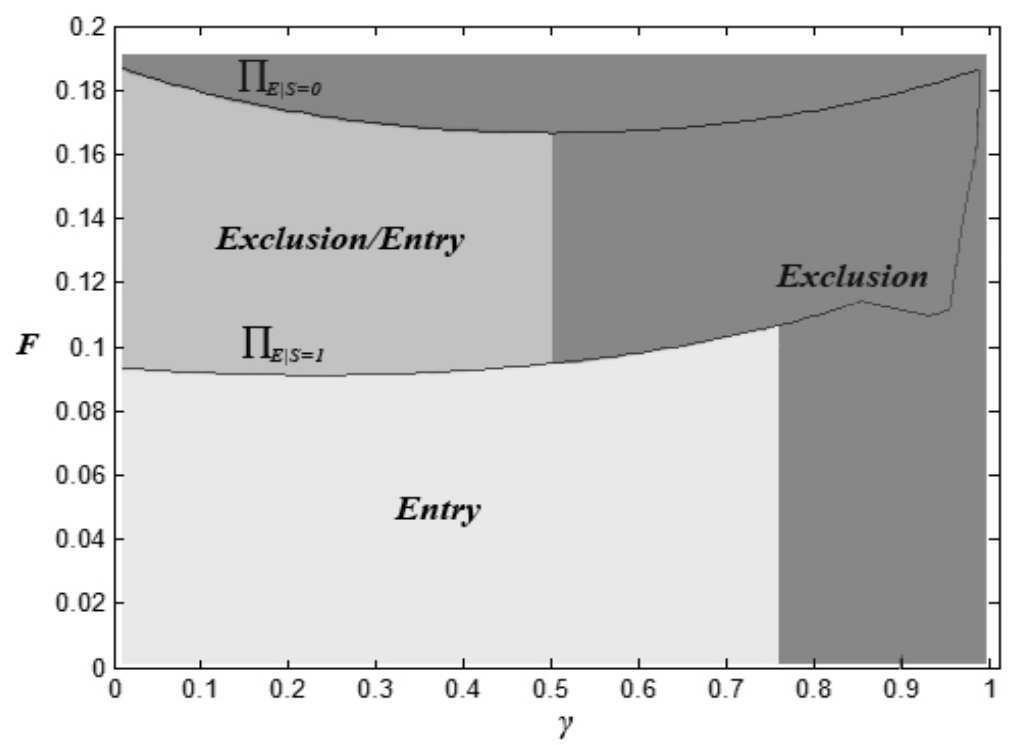

Figure 2 